\title{
Revealing Nanometre-scaled Solute Clusters in Neutron Irradiated Low Alloy Steels
}

\author{
Joven $\mathrm{J} \mathrm{H} \mathrm{Lim}^{1}$ and M Grace Burke ${ }^{2}$ \\ 1. Department of Theory and Modeling, United Kingdom Atomic Energy Authority, Culham Science \\ Centre, Abingdon OX14 3DB, UK \\ 2. The School of Materials, the University of Manchester, Oxford Road, Manchester M13 9PL, UK
}

The understanding of the formation of nanometre-scaled solute clusters that result in significant increases in yield strength of a reactor pressure vessel steel is crucial for the program of light water reactor sustainability. It has been a challenge to study the nanometre-scaled solute features in irradiated reactor pressure vessels steel, i.e. low alloy steel, using transmission electron microscope (TEM). This is due to multiple reasons. Two of the main reasons that have direct influences on detecting these nanometre-scaled solute features are (1) the ferromagnetic-nature of the low alloy steels and (2) the nature of these nanometre-scaled solute features. To minimise the influences of the former, i.e. ferromagnetic-nature of the low alloy steels, smaller TEM specimens were prepared using Focused Ion Beam (FIB). Even though there are FIB induced damages such as amorphous and irradiation-induced dislocation loops that are very similar to those induced by neutron irradiation in low alloy steels, it is possible to 'clean-up' these FIB damages, as shown in Figure 1. Further details of the 'cleaning' process will be discussed. The influence of the latter, i.e. the nature of these nanometre-scaled solute features, cannot be altered or improved. These nanometre-scaled solute clusters induced very low strain field to the surrounding matrix. It is very difficult to reveal these features even when using a weak-beam dark-field imaging condition [1].

In this study, three neutron irradiated model alloys to very high dpa, i.e. $\sim 1$ dpa, have been characterised using a FEI aberration-corrected Titan G2 80-200 S/TEM X-FEG with Super X EDX and a Gatan Quantum 965 EELS system. These alloys have a chemical composition that is similar to an A508-type commercial grade low alloy steels but have different concentrations of Nickel (Ni), Manganese (Mn) and Copper $(\mathrm{Cu})$, i.e. LA (0.4 wt.\% of $\mathrm{Cu}$ and 1.37 wt.\% of $\mathrm{Mn}), \mathrm{LD}(0.38 \mathrm{wt} . \%$ of $\mathrm{Cu}, 1.25 \mathrm{wt} . \%$ of $\mathrm{Ni}$ and 1.38 wt. $\%$ of $\mathrm{Mn}$ ) and CM6 (1.7 wt.\% of $\mathrm{Ni}$ and $1.5 \mathrm{wt} . \%$ of Mn). STEM-EELS analyses on these model alloys show that the nanometre-scaled solute features that nucleated and formed during the neutron irradiation are strongly dependent on the alloying elements; in this case, $\mathrm{Ni}, \mathrm{Mn}$ and $\mathrm{Cu}$. Figures 2, 3 and 4 show the HAADF micrographs and EELS reconstructed spectrum images (RSIs) of the solute clusters formed in the neutron irradiated LA, LD and CM6, respectively. It is important to note that without the presence of $\mathrm{Ni}$ in the alloy, $\mathrm{Mn}$ and $\mathrm{Si}$ were segregated to well-resolved dislocation loops, as shown in Figure 2, and no other nanometre-scaled solute features were observed. For the alloys with Ni, Ni-Mn-Sienriched solute clusters were detected as shown in Figures 3 and 4. The existence of dislocation loops in LD and CM6 and the comparison with modelling will be further discussed.

\section{References:}

[1] J J H Lim et al. (2013), "Microstructural Characterisation of Irradiation-Induced MnNi-Rich Solute Cluster in Highly Neutron-Irradiated MnNiMo Alloyed Weld Metals", 2013, ASTM International, West Conshohocken, PA. DOI: 10/152/STP157220130120

[2] Acknowledgements: Project sponsors - Rolls Royce UK, ATR-NSUF and CAES-MaCS. 


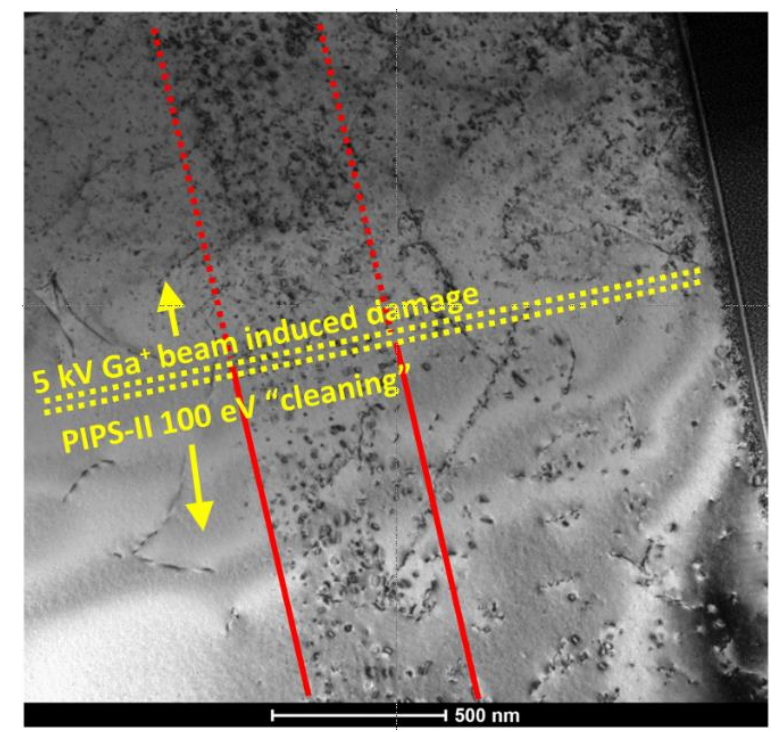

Figure 1. (Left) Bright-field kinematic (BFK) micrograph imaged from a FIB TEM specimen from a $5 \mathrm{MeV} \mathrm{Fe}^{2+}$ irradiated Alloy $800 \mathrm{H}$ that has been 'cleaned' using a post-FIB low energy ion beam polishing process. Due to the shadowing effect during the polishing process, FIB - damages induced during the TEM preparation remained, as shown in the upper half of the BFK micrograph. The lower half of the BFK micrograph showed an improved quality in the region where the specimen was 'cleaned' with low energy ion beams.

(2)
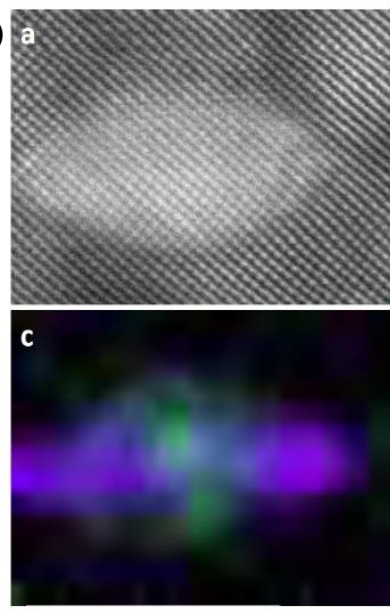

$5 \mathrm{~nm}$

(4)
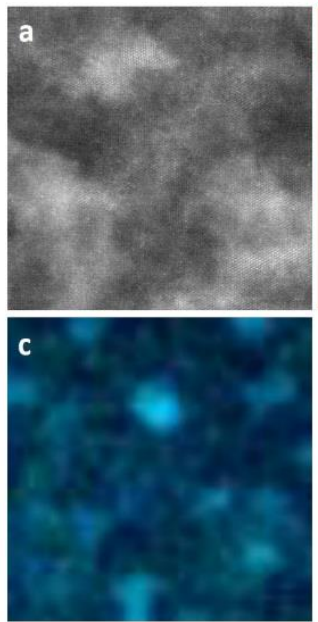

b

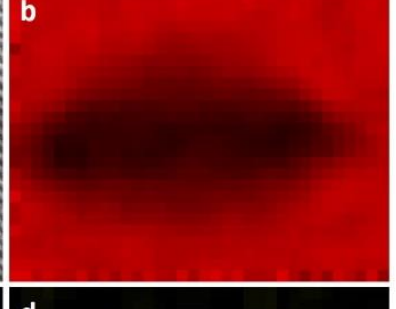

d

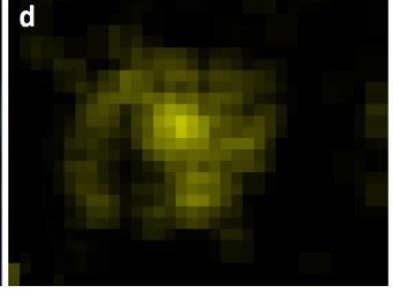

(3)
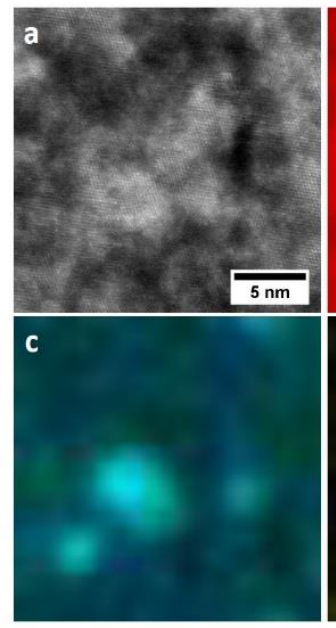

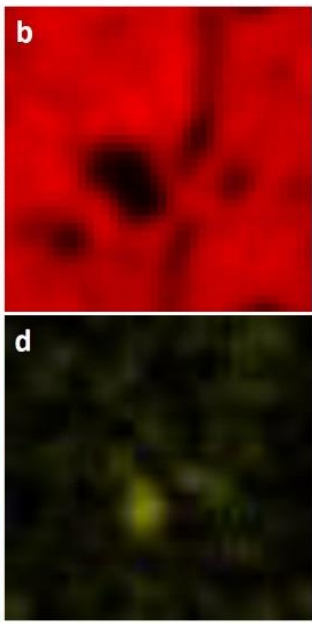

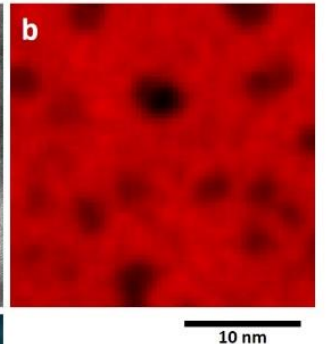

$10 \mathrm{~nm}$

Figure 2. (a) HAADF micrographs and multivariate statistical analyzed (MSA) Electron Energy Loss Spectroscopy (EELS) reconstructed spectrum images (RSI) of (b) Fe L-edge (red), (c) Mn L-edges (purple) and Cu Ledge (green), and $\mathrm{Cu}$ L-edge (yellow) showing a solute cluster formed in neutron irradiated LA.

Figure 3. (a) HAADF micrographs and MSA EELS RSI of (b) Fe L-edge (red), (c) Ni \& Mn L-edges (skyblue) and Cu L-edge (green), and $\mathrm{Cu}$ L-edge (yellow) showing solute clusters formed in neutron irradiated LD.

Figure 4. (a) HAADF micrographs and MSA EELS RSI of (b) Fe L-edge (red), (c) Ni \& Mn L-edges (skyblue) showing solute clusters formed in neutron irradiated CM6. 